\title{
Electronic cigarettes: the lesser of two evils, but how much less?
}

\author{
Steven D Shapiro, ${ }^{1}$ Ata Murat Kaynar ${ }^{2,3}$
}

No legal habit has caused more morbidity and mortality than cigarette smoking which takes greater than 10 years away from the average smoker's life, being a major risk factor for the leading causes of mortality in both developed and lowincome/middle-income countries, including coronary artery disease, cancers (especially lung cancer) and COPD. ${ }^{1-4}$ While cigarette smoking is increasing worldwide, we have made slow but steady progress in the USA reducing smoking prevalence to $18 \%$ of adults. Nicotine replacement therapy has assisted many smokers to quit; however, with the advent of electronic cigarettes (e-cigarettes), investigators began to wonder to what extent vaporised nicotine is responsible for disease pathogenesis beyond its addictive properties. Nicotine modulates inflammation causing both inflammatory cell apoptosis ${ }^{5}$ and inflammatory cell chemotaxis. ${ }^{6}$ There is growing evidence that e-cigarettes increase the risk for oxidative burden and inflammation in the lungs of mice. ${ }^{7}$ The study by Garcia-Arcos et $a l^{8}$ published in Thorax convincingly demonstrates that chronic exposure (4 months) of inhaled e-cigarettes in mice causes characteristic changes observed in COPD, including airway pathology, inflammation and emphysematous lung destruction.

Are preclinical models of COPD sufficient to consider regulating the use of e-cigarettes? Murine models of disease are often criticised for lack of translation to man, yet if we are cognizant of the strengths and shortcomings of each model, then logical extrapolations can be made. For example, chronic exposure to cigarette smoke in mice leads to acute neutrophil recruitment followed by macrophage accumulation and lung destruction with airspace enlargement that is very similar to human

\footnotetext{
'Department of Medicine, University of Pittsburgh School of Medicine and UPMC, Pittsburgh, Pennsylvania, USA; ${ }^{2}$ Department of Critical Care Medicine, University of Pittsburgh School of Medicine and UPMC, Pittsburgh, Pennsylvania, USA; ${ }^{3}$ The Clinical Research, Investigation, and Systems Modeling of Acute Illness (CRISMA) Center, University of Pittsburgh School of Medicine and UPMC, Pittsburgh, Pennsylvania, USA
}

Correspondence to Dr Steven D Shapiro, Department of Medicine, University of Pittsburgh School of Medicine, U.S. Steel Tower, 600 Grant Street, Suite 6250, Pittsburgh, PA 15219, USA; shapirosd@upmc.edu emphysema. Mice deficient in macrophage elastase (MMP-12) were entirely protected from cigarette smoke-induced emphysema. ${ }^{9}$ This highlighted the role of macrophages in emphysema, although it was thought that other macrophage proteases such as MMP-9 play a larger role in humans. Yet, a recent meta-analysis of COPD genomewide association study (GWAS) studies identified MMP-12 as one of three genes of significance, highlighting the utility of animals to model aspects of human disease. ${ }^{10}$ In the current study, e-cigarettes led to a very 'COPD-like' inflammatory-destructive profile with macrophage dominant cellular influx in the bronchoalveolar lavage and proinflammatory cytokines (interleukin (IL)-1 $\beta$, monocyte chemoattractant protein (MCP)-1, chemokine (C-X- C motif) ligand (CXCL)10, IL-6, CXCL2 and CXCL5) and proteases MMP-3, MMP-9, MMP-12, Cathepsin K and Cathepsin L1.

Modelling small airway disease of COPD in mice has been more difficult. Anatomically, mouse airways have much less branching points and no respiratory bronchioles, the initial site of narrowing in COPD. In addition, it has been difficult to show significant hyper-reactivity in response to cigarette smoke alone without addition of ovalbumin. Nevertheless, one can observe some pathological changes such as airway fibrosis and periodic acidSchiff (PAS) + staining with smoking. In the current study, e-cigarettes resulted in increased airway resistance without sensitisation, perhaps modelling human COPD better than real cigarettes. To further model the airway in COPD, the group applied nicotine to human epithelial cells on an air-liquid interface. While difficult to translate studies using 'liquid smoke' or in this case e-cigarette smoke directly on cells, these studies were well at least well controlled and the findings are interesting showing ciliary movement suppression, Cystic Fibrosis Transmembrane Conductance Regulator (CFTR) dysfunction and downregulation of the big potassium (BK) channel alpha subunit KCNMA1. These findings are observed in human COPD reducing bacterial clearance, causing surface liquid dehydration and increasing airway viscosity, respectively.

The authors did not compare e-cigarettes with true cigarettes in their model (normalised for nicotine content), so we do not know the full extent to which nicotine accounts for cigarette smoke-induced emphysema. Nevertheless, the study strongly suggests that e-cigarettes have direct deleterious effects beyond their addictive capacity. How much human translation is necessary to take action? Assuming one observes macrophage accumulation and activation in human bronchoalveolar lavage from e-cigarette users, then it follows that lung destruction would follow, in at least some patients.

What then could be done with this information? Recently, in May 2016, the 'deeming rule' was passed giving the US Food and Drug Administration (FDA) the power to retrospectively regulate tobacco products that were recently introduced into the market, including e-cigarettes and hookahs. ${ }^{11}$ How then should this new power be exerted with respect to ecigarette regulation? It is tempting to remove e-cigarettes entirely, but let's take a closer look.

1. Even if this single substance nicotine (and minimal number of other particles in this vaporised product) did cause the majority of COPD, it must certainly be less harmful overall than the myriad of particulates, including carcinogens, emitted from cigarettes and is most likely the lesser of two evils.

2. In the UK over the past 10 years, the availability of e-cigarettes correlated with reduction in cigarette smoking with quit rates increasing from $11 \%$ to $19 \%$ as e-cigarettes grew from essentially $0 \%$ to $21 \%$ (while other nicotine replacement therapies decreased significantly). ${ }^{12}$ Given the potential to increase smoking cessation, perhaps they should be discouraged entirely in non-smokers and as a long-term replacement for cigarettes, but serve as a temporary bridge to smoking cessation.

Theoretically, stepwise reduction in inflammation might actually be the preferred way to quit. For example, while MMP-12 is destructive to lung tissue, it aids the host in limiting tumour cell growth and angiogenesis. ${ }^{13}$ The fear is that quitting cold turkey would limit lung destruction but allow small nests of tumour cells to grow. A preliminary observation from our University of Pittsburgh Medical Center (UPMC) population demonstrated that the overall risk of developing lung cancer in our COPD population is $<2 \%$, but was $5 \%$ during the first year of quitting (Shapiro, unpublished). While only a simple observation with multiple potential explanations, it may suggest that the gradual reduction in 
inflammation-e-cigarettes for a short time before total discontinuation-a plausible and testable hypothesis. For some, smoking cessation is not possible, and long-term e-cigarettes preferred over cigarette smoking. Like methadone for opioid addiction, e-cigarettes may be a necessary long-term substitute for smokers. ${ }^{14-17}$

Contributors Both authors contributed to the Editorial.

Funding National Heart, Lung, and Blood Institute (HL126711).

Competing interests None declared.

Provenance and peer review Commissioned; externally peer reviewed.

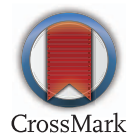

To cite Shapiro SD, Kaynar AM. Thorax 2016;71:1080-1081.

\section{SLinked}

http://dx.doi.org/10.1136/thoraxjnl-2015-208039

Thorax 2016:71:1080-1081.

doi:10.1136/thoraxjnl-2016-209273

\section{REFERENCES}

1 Jha P, Ramasundarahettige C, Landsman V, et al. 21st-century hazards of smoking and benefits of cessation in the United States. N Engl J Med 2013;368:341-50.

2 Kontis V, Mathers CD, Rehm J, et al. Contribution of six risk factors to achieving the $25 \times 25$ non-communicable disease mortality reduction target: a modelling study. Lancet 2014;384: 427-37.

3 Zheng W, McLerran DF, Rolland BA, et al. Burden of total and cause-specific mortality related to tobacco smoking among adults aged $\geq 45$ years in Asia: a pooled analysis of 21 cohorts. PLoS Med 2014;11: e1001631.

4 Critchley JA, Capewell S. Mortality risk reduction associated with smoking cessation in patients with coronary heart disease: a systematic review. JAMA 2003;290:86-97.

5 Mariggiò MA, Guida L, Laforgia A, et al. Nicotine effects on polymorphonuclear cell apoptosis and lipopolysaccharide-induced monocyte functions. A possible role in periodontal disease? J Periodont Res 2001;36:32-9.

6 Totti N III, McCusker KT, Campbell EJ, et al. Nicotine is chemotactic for neutrophils and enhances neutrophil responsiveness to chemotactic peptides. Science 1984;223:169-71.

7 Lerner CA, Sundar IK, Yao H, et al. Vapors produced by electronic cigarettes and e-juices with flavorings induce toxicity, oxidative stress, and inflammatory response in lung epithelial cells and in mouse lung. PLOS ONE 2015;10:e0116732.

8 Garcia-Arcos I, Geraghty P, Baumlin N, et al. Chronic electronic cigarette exposure in mice induces COPD in a nicotine-dependent manner. Thorax 2016;71:1119-29.
9 Hautamaki RD, Kobayashi DK, Senior RM, et al. Requirement for macrophage elastase for cigarette smoke-induced emphysema in mice. Science 1997:277:2002-4.

10 Cho MH, McDonald ML, Zhou X, et al. Risk loci for chronic obstructive pulmonary disease: a genome-wide association study and meta-analysis. Lancet Respir Med 2014;2:214-15.

11 U.S Food and Drug Administration. Deeming tobacco products to be subject to the Federal Food, Drug, and Cosmetic Act, as amended by the Family Smoking Prevention and Tobacco Control Act. 21 CFR Part 1100,1140, and 1143. Silver Spring, MD: U.S Food and Drug Administration, May 2016. http:// www.fda.gov/TobaccoProductcs/Labeling/ RulesRegulationsGuidance/ucm394909.htm

12 Britton J. Electronic cigarettes and smoking cessation in England. BMJ 2016;354:i4819.

13 Houghton AM, Grisolano JL, Baumann ML, et al. Macrophage elastase (MMP-12) suppresses growth of lung metastases. Cancer Res 2006;66:6149-55.

14 Huen KH, Chowdhury R, Shafii SM, et al. Smoking cessation is the least successful outcome of risk factor modification in uninsured patients with symptomatic peripheral arterial disease. Ann Vasc Surg 2015;29:42-9.

15 Smith SS, Fiore MC, Baker TB. Smoking cessation in smokers who smoke menthol and non-menthol cigarettes. Addiction 2014;109:2107-17.

16 Chen LS, Baker TB, Piper ME, et al. Interplay of genetic risk factors (CHRNA5-CHRNA3-CHRNB4) and cessation treatments in smoking cessation success. Am J Psychiatry 2012;169:735-42.

17 Kenford SL, Fiore MC, Jorenby DE, et al. Predicting smoking cessation. Who will quit with and without the nicotine patch. JAMA 1994;271:589-94. 